\title{
Burden of Transport-Related Injuries in the Eastern Mediterranean Region: A Systematic Analysis for the Global Burden of Disease Study 2017
}

GBD 2017 EMR Transport Injuries Collaborators, Saeid Safiri, PhD ${ }^{1,2^{*}}$; Mark J. M. Sullman, PhD ${ }^{3,4}$; Timo Lajunen, PhD ${ }^{5}$;etiana Hill, PhD' ; Amir Almasi-Hashiani, PhD'; Maziar Moradi-Lakeh, MD ${ }^{8}$; Farshad Farzadfar, MD $^{9}$; Sadaf G. Sepanlou, MD ${ }^{10,11}$; Eman Abu-Gharbieh, PhD ${ }^{12}$; Teamur Aghamolaei, PhD ${ }^{13}$; Tauseef Ahmad, PhD ${ }^{14}$; Suliman A. Alghnam, PhD ${ }^{15,16}$; Samar Al-Hajj, PhD ${ }^{17,18}$; Vahid Alipour, PhD ${ }^{19,20}$; Syed Mohamed Aljunid, MD, PhD ${ }^{21,22}$; Mina Anjomshoa, PhD ${ }^{23}$; Alireza Ansari-Moghaddam, PhD ${ }^{24}$; Jalal Arabloo, PhD ${ }^{19}$; Mohsen Bayati, PhD ${ }^{25}$; Neeraj Bedi, MD $^{26,27}$; Salaheddine Bendak, PhD ${ }^{28,29}$; Zulfiqar A Bhutta, PhD ${ }^{30,31}$; Ali Bijani, PhD ${ }^{32}$; Saad M A Dahlawi, PhD ${ }^{33}$; Mostafa Dianatinasab, MSc ${ }^{34,35}$; Zahra Sadat Dibaji Forooshani, PhD ${ }^{36}$; Hala Rashad Elhabashy, MD ${ }^{37}$; Amir Emami Zeydi, PhD ${ }^{38}$; Sharareh Eskandarieh, $\mathrm{PhD}^{39}$; Mansour Ghafourifard, $\mathrm{PhD}^{40}$; Ahmad Ghashghaee, BSc $^{19,41}$; Michal Grivna, MD, PhD ${ }^{42,43}$; Mohammed Ibrahim Mohialdeen Gubari, PhD ${ }^{44}$; Randah R Hamadeh, PhD ${ }^{45}$; Samer Hamidi, $\mathrm{DrPH}^{46}$; Khezar Hayat, MSc ${ }^{47,48}$; Enayatollah Homaie Rad, PhD ${ }^{49,50}$; Mehdi Hosseinzadeh, PhD ${ }^{51,52}$; Mowafa Househ, PhD ${ }^{53}$; Seyed Sina Naghibi Irvani, $\mathbf{M D}^{54}$; Mohammad Ali Jahani, $\mathrm{PhD}^{32}$; Leila R Kalankesh, PhD ${ }^{55}$; Rohollah Kalhor, PhD ${ }^{56,57}$; Ibrahim Kamel, $\mathrm{MD}^{58,59}$; Mohammad Khammarnia, $\mathrm{PhD}^{60}$; Maseer Khan, $\mathrm{MD}^{61}$; Habibolah Khazaie, $\mathrm{MD}^{62}$; Hamidreza Komaki, $\mathrm{MD}^{63,64}$; Amitis Lahimchi, BDent ${ }^{65}$; Mohammed Madadin, MD ${ }^{66}$; Shokofeh Maleki, MSc ${ }^{67}$; Navid Manafi, MD $^{68,69}$; Fariborz Mansour-Ghanaei, MD $^{70,71}$; Mohammad Ali Mansournia, MD, PhD ${ }^{72}$; Ritesh G Menezes, $\mathbf{M D}^{73}$; Yousef Mohammad, $\mathbf{M D}^{74}$; Abdollah MohammadianHafshejani, PhD"75; Farnam Mohebi, MD' ${ }^{9,76}$; Ghobad Moradi, MD, PhD ${ }^{77,78}$; Rahmatollah Moradzadeh, PhD ${ }^{7}$; Seyyed Meysam Mousavi, PhD ${ }^{79}$; Mehdi Naderi, PhD ${ }^{67}$; Rajan Nikbakhsh, MD $^{80}$; Keyvan Pakshir, PhD ${ }^{81}$; Akram Pourshams, MD $^{10}$; Navid Rabiee, MSc $^{82}$; Alireza Rafiei, $\mathrm{PhD}^{83}{ }^{84}$; Reza Rawassizadeh, $\mathrm{PhD}^{85}$; Aziz Rezapour, $\mathrm{PhD}^{19}$; Basema Saddik, $\mathrm{PhD}^{86}$; Sahar Saeedi Moghaddam, MSc $^{9}$; Payman Salamati, MD ${ }^{87}$; Marwa Rashad Salem, MD ${ }^{88}$; Hosni Salem, MD ${ }^{89}$; Abdallah M Samy, PhD ${ }^{90}$; Brijesh Sathian, PhD ${ }^{91}$ 92; Saeed Shahabi, PhD ${ }^{93}$; Masood Ali Shaikh, MD ${ }^{94}$; Mehran Shams-Beyranvand, MSc ${ }^{95}$; Morteza Shamsizadeh, MSc ${ }^{96}$; Mohammad Reza Sobhiyeh, MD ${ }^{97}$; Amin Soheili, PhD ${ }^{98}$; Arash Tehrani-Banihashemi, PhD ${ }^{8,99}$; Yasir Waheed, PhD ${ }^{100}$; Hasan Yusefzadeh, PhD ${ }^{101}$; Telma Zahirian Moghadam, PhD ${ }^{102}$; Leila Zaki, PhD ${ }^{103}$; Mohammad Zamani, MD ${ }^{104}$; Hamed Zandian, PhD ${ }^{102,105}$; Reza Malekzadeh, $\mathrm{MD}^{10,11}$; and Mohsen Naghavi, MD, PhD ${ }^{106,107^{*}}$

${ }^{1}$ Road Traffic Injury Research Center, Tabriz University of Medical Sciences, Tabriz, Iran

${ }^{2}$ Department of Community Medicine, Faculty of Medicine, Tabriz University of Medical Sciences, Tabriz, Iran

${ }^{3}$ Department of Social Sciences, University of Nicosia, Nicosia, Cyprus

${ }^{4}$ Department of Life and Health Sciences, University of Nicosia, Nicosia, Cyprus

${ }^{5}$ Department of Psychology, Norwegian University of Science and Technology, Trondheim, Norway

${ }^{6}$ Hertfordshire Business School, University of Hertfordshire, Hatfield, UK

'Department of Epidemiology, Arak University of Medical Sciences, Arak, Iran

${ }^{8}$ Preventive Medicine and Public Health Research Center, Iran University of Medical Sciences, Tehran, Iran

${ }^{9}$ Non-communicable Diseases Research Center, Tehran University of Medical Sciences, Tehran, Iran

${ }^{10}$ Digestive Diseases Research Institute, Tehran University of Medical Sciences, Tehran, Iran

${ }^{11}$ Non-communicable Disease Research Center, Shiraz University of Medical Sciences, Shiraz, Iran

${ }^{12}$ Department of Clinical Sciences, University of Sharjah, Sharjah, United Arab Emirates

${ }^{13}$ Department of Health Education and Health Promotion, Hormozgan University of Medical Sciences, Bandar Abbas, Iran

${ }^{14}$ Department of Epidemiology and Health Statistics, Southeast University, Nanjing, China

${ }^{15}$ Population Health Research Section, King Abdullah International Medical Research Center, Riyadh, Saudi Arabia

${ }^{16}$ King Abdullah International Medical Research Center, King Saud bin Abdulaziz University for Health Sciences, Riyadh, Saudi Arabia

${ }^{17}$ Faculty of Health Sciences, American University of Beirut, Beirut, Lebanon

${ }^{18}$ British Columbia Injury Research Prevention Unit, British Columbia Children's Hospital Research Institute, Vancouver, BC, Canada

${ }^{19} \mathrm{Health}$ Management and Economics Research Center, Iran University of Medical Sciences, Tehran, Iran

${ }^{20}$ Department of Health Economics, Iran University of Medical Sciences, Tehran, Iran

${ }^{21}$ Department of Health Policy and Management, Kuwait University, Kuwait City, Kuwait

${ }^{22}$ International Centre for Casemix and Clinical Coding, National University of Malaysia, Bandar Tun Razak, Malaysia

${ }^{23}$ Social Determinants of Health Research Center, Rafsanjan University of Medical Sciences, Rafsanjan, Iran

${ }^{24}$ Department of Epidemiology and Biostatistics, Zahedan University of Medical Sciences, Zahedan, Iran

${ }^{25}$ Health Human Resources Research Center, Shiraz University of Medical Sciences, Shiraz, Iran

${ }^{26}$ Department of Community Medicine, Gandhi Medical College Bhopal, Bhopal, India

${ }^{27}$ Jazan University, Jazan, Saudi Arabia

*Corresponding Author: Saeid Safiri, PhD, Road Traffic Injury Research Center, Tabriz University of Medical Sciences, Tabriz, Iran; Department of Community Medicine, Faculty of Medicine, Tabriz University of Medical Sciences, Tabriz, Iran. Tel: 0098-4133364673; Email: safiris@tbzmed.ac.ir Mohsen Naghavi, MD, PhD; Institute for Health Metrics and Evaluation, University of Washington, Seattle, WA, USA; Department of Health Metrics Sciences, School of Medicine, University of Washington, Seattle, WA, USA. Tel: 001- 2068972800; Emails: nagham@uw.edu 
${ }^{28}$ Department of Industrial Engineering and Engineering Management, University of Sharjah, Sharjah, United Arab Emirates

${ }^{29}$ Department of Epidemiology, University of Sharjah, Sharjah, United Arab Emirates

${ }^{30}$ Centre for Global Child Health, University of Toronto, Toronto, ON, Canada

${ }^{31}$ Centre of Excellence in Women \& Child Health, Aga Khan University, Karachi, Pakistan

${ }^{32}$ Social Determinants of Health Research Center, Babol University of Medical Sciences, Babol, Iran

${ }^{33}$ Environmental Health Department, Imam Abdulrahman Bin Faisal University, Dammam, Saudi Arabia

${ }^{34}$ Department of Epidemiology and Biostatistics, Shahroud University of Medical Sciences, Shahroud, Iran

${ }^{35}$ Department of Epidemiology, Shiraz University of Medical Sciences, Shiraz, Iran

${ }^{36}$ Tehran University of Medical Sciences, Tehran, Iran

${ }^{37}$ Neurophysiology Department, Cairo University, Cairo, Egypt

${ }^{38}$ Department of Medical-Surgical Nursing, Mazandaran University of Medical Sciences, Sari, Iran

${ }^{39}$ Multiple Sclerosis Research Center, Tehran University of Medical Sciences, Tehran, Iran

${ }^{40}$ Department of Medical Surgical Nursing, Tabriz University of Medical Sciences, Tabriz, Iran

${ }^{41}$ Student Research Committee, Iran University of Medical Sciences, Tehran, Iran

${ }^{42}$ Institute of Public Health, United Arab Emirates University, Al Ain, United Arab Emirates

${ }^{43}$ Department of Public Health and Preventive Medicine, Charles University, Prague, Czech Republic

${ }^{44}$ Department of Family and Community Medicine, University of Sulaimani, Sulaimani, Iraq

${ }^{45}$ Department of Family and Community Medicine, Arabian Gulf University, Manama, Bahrain

${ }^{46}$ School of Health and Environmental Studies, Hamdan Bin Mohammed Smart University, Dubai, United Arab Emirates

${ }^{47}$ Institute of Pharmaceutical Sciences, University of Veterinary and Animal Sciences, Lahore, Pakistan

${ }^{48}$ Department of Pharmacy Administration and Clinical Pharmacy, Xian Jiaotong University, Xian, China

${ }^{49}$ Guilan Road Trauma Research Center, Guilan University of Medical Sciences, Rasht, Iran

${ }^{50}$ Social Determinants of Health Research Center, Guilan University of Medical Sciences, Rasht, Iran

${ }^{51}$ Institute of Research and Development, Duy Tan University, Da Nang, Vietnam

${ }^{52}$ Department of Computer Science, University of Human Development, Sulaymaniyah, Iraq

${ }^{53}$ College of Science and Engineering, Hamad Bin Khalifa University, Doha, Qatar

${ }^{54}$ Research Institute for Endocrine Sciences, Shahid Beheshti University of Medical Sciences, Tehran, Iran

${ }^{55}$ Health Services Management Research Center, Tabriz University of Medical Sciences, Tabriz, Iran

${ }^{56}$ Institute for Prevention of Non-communicable Diseases, Qazvin University of Medical Sciences, Qazvin, Iran

${ }^{57}$ Health Services Management Department, Qazvin University of Medical Sciences, Qazvin, Iran

${ }^{58}$ University of La Verne, La Verne, CA, USA

${ }^{59}$ Department of Medicine, October 6 University, Giza, Egypt

${ }^{60}$ Health Promotion Research Center, Zahedan University of Medical Sciences, Zahedan, Iran

${ }^{61}$ Epidemiology Department, Jazan University, Jazan, Saudi Arabia

${ }^{62}$ Department of Psychiatry, Kermanshah University of Medical Sciences, Kermanshah, Iran

${ }^{63}$ Neurophysiology Research Center, Hamadan University of Medical Sciences, Hamadan, Iran

${ }^{64}$ Brain Engineering Research Center, Institute for Research in Fundamental Sciences, Tehran, Iran

${ }^{65}$ First Faculty of Medicine, Charles University, Prague, Czech Republic

${ }^{66}$ Pathology Department, Imam Abdulrahman Bin Faisal University, Dammam, Saudi Arabia

${ }^{67}$ Clinical Research Development Center, Kermanshah University of Medical Sciences, Kermanshah, Iran

${ }^{68} \mathrm{~S}$ chool of Medicine, Iran University of Medical Sciences, Tehran, Iran

${ }^{69}$ School of Medicine, University of Manitoba, Winnipeg, MB, Canada

${ }^{70}$ Gastrointestinal and Liver Diseases Research Center, Guilan University of Medical Sciences, Rasht, Iran

${ }^{71}$ Caspian Digestive Disease Research Center, Guilan University of Medical Sciences, Rasht, Iran

${ }^{72}$ Department of Epidemiology and Biostatistics, Tehran University of Medical Sciences, Tehran, Iran

${ }^{73}$ Forensic Medicine Division, Imam Abdulrahman Bin Faisal University, Dammam, Saudi Arabia

${ }^{74}$ Internal Medicine Department, King Saud University, Riyadh, Saudi Arabia

${ }^{75}$ Department of Epidemiology and Biostatistics, Shahrekord University of Medical Sciences, Shahrekord, Iran

${ }^{76}$ National Institute of Health Research (NIHR), Tehran University of Medical Sciences, Tehran, Iran

${ }^{77}$ Social Determinants of Health Research Center, Kurdistan University of Medical Sciences, Sanandaj, Iran

${ }^{78}$ Department of Epidemiology and Biostatistics, Kurdistan University of Medical Sciences, Sanandaj, Iran

${ }^{79}$ Department of Health Policy, Management, and Economics, Tehran University of Medical Sciences, Tehran, Iran

${ }^{80}$ Obesity Research Center, Shahid Beheshti University of Medical Sciences, Tehran, Iran

${ }^{81}$ Department of Parasitology and Mycology, Shiraz University of Medical Sciences, Shiraz, Iran

${ }^{82}$ Department of Chemistry, Sharif University of Technology, Tehran, Iran

${ }^{83}$ Department of Immunology, Mazandaran University of Medical Sciences, Sari, Iran

${ }^{84}$ Molecular and Cell Biology Research Center, Mazandaran University of Medical Sciences, Sari, Iran

${ }^{85}$ Department of Computer Science, Boston University, Boston, MA, USA

${ }^{86}$ Department of Family and Community Medicine, University of Sharjah, Sharjah, United Arab Emirates

${ }^{87}$ Sina Trauma and Surgery Research Center, Tehran University of Medical Sciences, Tehran, Iran

${ }^{88}$ Public Health and Community Medicine Department, Cairo University, Giza, Egypt

${ }^{8}$ Urology Department, Cairo University, Cairo, Egypt

${ }^{90}$ Department of Entomology, Ain Shams University, Cairo, Egypt

${ }^{91}$ Department of Geriatrics and Long Term Care, Hamad Medical Corporation, Doha, Qatar 
${ }^{92}$ Faculty of Health and Social Sciences, Bournemouth University, Bournemouth, UK

${ }^{93}$ Health Policy Research Center, Shiraz University of Medical Sciences, Shiraz, Iran

${ }^{94}$ Independent Consultant, Karachi, Pakistan

${ }^{95}$ School of Medicine, Alborz University of Medical Sciences, Karaj, Iran

${ }^{96}$ Faculty of Caring Science, Work Life, and Social Welfare, University of Borås, Borås, Sweden

${ }^{97}$ Department of Vascular and Endovascular Surgery, Kermanshah University of Medical Sciences, Kermanshah, Iran

${ }^{98}$ Nursing Care Research Center, Semnan University of Medical Sciences, Semnan, Iran

${ }^{99}$ Department of Community and Family Medicine, Iran University of Medical Sciences, Tehran, Iran

${ }^{100}$ Foundation University Medical College, Foundation University Islamabad, Islamabad, Pakistan

${ }^{101}$ Department of Health Care Management and Economics, Urmia University of Medical Science, Urmia, Iran

${ }^{102}$ Social Determinants of Health Research Center, Ardabil University of Medical Science, Ardabil, Iran

${ }^{103}$ Department of Parasitology and Entomology, Tarbiat Modares University, Tehran, Iran

${ }^{104}$ Student Research Committee, Babol University of Medical Sciences, Babol, Iran

${ }^{105}$ Department of Community Medicine, Ardabil University of Medical Science, Ardabil, Iran

${ }^{106}$ Institute for Health Metrics and Evaluation, University of Washington, Seattle, WA, USA

${ }^{107}$ Department of Health Metrics Sciences, School of Medicine, University of Washington, Seattle, WA, USA

\begin{abstract}
Background: Transport-related injuries (TIs) are a substantial public health concern for all regions of the world. The present study quantified the burden of Tls and deaths in the Eastern Mediterranean region (EMR) in 2017 by sex and age.

Methods: Tls and deaths were estimated by age, sex, country, and year using Cause of Death Ensemble modelling (CODEm) and DisMod-MR 2.1. Disability-adjusted life years (DALYs), which quantify the total burden of years lost due to premature death or disability, were also estimated per 100000 population. All estimates were reported along with their corresponding $95 \%$ uncertainty intervals (UIs).

Results: In 2017, there were 5.5 million (UI 4.9-6.2) transport-related incident cases in the EMR - a substantial increase from 1990 (2.8 million; UI 2.5-3.1). The age-standardized incidence rate for the EMR in 2017 was 787 (UI 705.5-876.2) per 100 000, which has not changed significantly since 1990 (-0.9\%; UI -4.7 to 3 ). These rates differed remarkably between countries, such that Oman (1303.9; UI 1167.3-1441.5) and Palestine (486.5; UI 434.5-545.9) had the highest and lowest age-standardized incidence rates per 100000 , respectively. In 2017, there were 185.3 thousand (UI 170.8-200.6) transport-related fatalities in the EMR - a substantial increase since 1990 (140.4 thousand; UI 118.7-156.9). The age-standardized death rate for the EMR in 2017 was 29.5 (UI 27.1-31.9) per 100000, which was 30.5\% lower than that found in 1990 (42.5; UI 36.8-47.3). In 2017, Somalia (54; UI 30-77.4) and Lebanon (7.1; UI 4.8-8.6) had the highest and lowest age-standardized death rates per 100,000, respectively. The age-standardised DALY rate for the EMR in 2017 was 1,528.8 (UI 1412.5-1651.3) per 100 000, which was 34.4\% lower than that found in 1990 (2,331.3; UI 1,993.1-2,589.9). In 2017, the highest DALY rate was found in Pakistan (3 454 121; UI 2297 8904342 908) and the lowest was found in Bahrain (8616; UI 7670-9751).

Conclusion: The present study shows that while road traffic has become relatively safer (measured by deaths and DALYs per 100000 population), the number of transport-related fatalities in the EMR is growing and needs to be addressed urgently.

Keywords: Accidents, Epidemiology, Incidence, Mortality, Road traffic injuries

Cite this article as: GBD 2017 EMR Transport Injuries Collaborators, Safiri S, Sullman MJM, Lajunen T, Hill T, Almasi-Hashiani A, et al. Burden of Transport-related Injuries in the Eastern Mediterranean Region: A Systematic Analysis for the Global Burden of Disease Study 2017. Arch Iran Med. 2021;24(7):512-525. doi: 10.34172/aim.2021.74
\end{abstract}

Received: September 13, 2020, Accepted: January 13, 2021, ePublished: July 1, 2021

\section{Introduction}

Transport-related injuries (TIs) are a major public health concern for all regions of the world. ${ }^{1}$ In 2016, the World Health Organization (WHO) reported that TIs were the eighth most common cause of death for people of all ages, accounting for more than $2.5 \%$ of all deaths worldwide. ${ }^{1}$ In the same year, TIs resulted in approximately 1.35 million deaths and were the single largest killer of children and young adults (5-29 years old). ${ }^{2}$ In comparison to 2015 , the number of deaths decreased globally, which suggests some progress in legislation and the accessibility of emergency medical services. ${ }^{2}$ However, it is highly unlikely that the United Nations Sustainable Development Goals target of halving road traffic deaths by 2020 globally can be met. ${ }^{2}$ In addition, most of the global reduction has been made in high-income countries, while the number of deaths in low to middle income countries has grown since $2013 .^{2}$

In 2016, the Eastern Mediterranean region (EMR) ranked the third highest in the world for TIs, with 18 road traffic deaths per 100000 people, surpassed only by Southeast Asia (20.7 per 100000) and Africa (26.6 per 100000). ${ }^{2}$ Furthermore, apart from being a major public health concern, TIs cost the region's economy billions of dollars each year. ${ }^{3}$ The EMR is composed of 22 countries from the Middle East, North Africa, and the Horn of Africa, with almost 710 million people living in this region. ${ }^{4}$ For the last decade, the EMR's economy has grown rapidly, which has also accelerated the development of road infrastructure and motorization. ${ }^{5}$ However, this has not positively affected the rates of transport-related mortality and morbidity in all countries in the region. ${ }^{6}$ On the contrary, while analyzing the burden of TIs in the EMR from 1990 to 2015, Khalil and colleagues concluded that death rates have increased in countries like Libya, Pakistan, and Egypt. ${ }^{6}$ Analyses of other country-specific data suggest that in Oman and the United Arab Emirates (UAE), the total road transport injury mortality was higher in 2016 than the year before. ${ }^{7}$ 
The road safety issues facing the EMR are not entirely due to the fact that many of the constituent countries are low- to middle-income countries. ${ }^{7}$ According to the WHO (2018), the EMR is the only region in the world where the increase in income does not lead to a decrease in the rate of road traffic deaths. ${ }^{2}$ The EMR also suffers from a lack of recent and reliable information regarding the current and long-term trends, which should be used to guide policymakers. ${ }^{6}$ In this study, we examined the burden of TIs in the EMR in 2017, by sex and age, through calculating disability-adjusted life years (DALYs) and the total numbers of TI-related incidence and fatalities. In addition, we calculated the associations between agestandardized DALYs and the Socio-demographic Index (SDI).

\section{Materials and Methods}

The Global Burden of Disease (GBD) study is the most global and wide-ranging research program on the burden of disease, with annual estimates. GBD 2017 included estimates for seven super-regions, 21 regions and 195 countries, from 1990 to 2017. There were 359 diseases and injuries, 282 causes of death, and 84 risk factors included in this iteration of the GBD. ${ }^{8-10}$ TIs included injuries incurred as pedestrians, cyclists, motorcyclists, motor vehicle occupants or drivers, as well as water and air transport. In this paper we report the burden of TIs for the 22 countries in the EMR region: Afghanistan, Bahrain, Djibouti, Egypt, Iran, Iraq, Jordan, Kuwait, Lebanon, Libya, Morocco, Oman, Pakistan, Palestine, Qatar, Saudi Arabia, Somalia, Sudan, Syria, Tunisia, the UAE, and Yemen.

\section{Case Definition}

Cases were identified using the International Classification of Diseases (ICD). ICD-9 codes E000-E999 and ICD-10 chapter $\mathrm{V}$ were used to define TI incidence and deaths. For more details, please consult previous research. ${ }^{8}$

\section{Fatal Injuries}

Input Data

In GBD 2017, the injury and mortality estimates were calculated using census data, verbal autopsy reports, vital or civil registration, surveys, mortality surveillance, and information from the police. Police reports were used to estimate the number of mortalities from road traffic injuries. ${ }^{8}$ The data collected from the police were obtained from national agencies, published research, and institutional surveys including the United Nations Crime Trends Survey and the WHO Global Status Report on Road Safety. Police records were not used in the countries with vital registration, unless the reported number of deaths in police records was higher than that recorded in vital registration. Outlier data sources were excluded from the study. ${ }^{8}$

\section{Modelling Strategy}

The GBD list of disease and injury causes was used to map all data sources. Following this, age-sex splitting was conducted, garbage codes were redistributed, and noise reduction processes were undertaken. The Cause of Death Ensemble modelling (CODEm) software was then used to compute estimates by year, EMR country, cause, sex, and age. Then, fatal discontinuity estimations for motor vehicle road injuries and other TIs were merged with the CODEm results, after the final cause of death results were calculated using Cause of Death Correct (CoDCorrect) ${ }^{8}$. CoDCorrect scales all cause-specific deaths from all causes of death for each group (i.e., sex, age group, year, and country) using an algorithm which makes certain that the sum matches the total for all-cause mortality. ${ }^{8}$ Further details regarding how the cause of death data were prepared, splitting the causes into sublevels, the model assumptions, the limitations of this approach, and the covariates used can be found in a previous publication. ${ }^{8}$

\section{Non-fatal Injuries \\ Input data}

Data from hospitals, emergency departments and insurance claims were used to estimate morbidity. Information from surveys were used to produce years lived with disability (YLDs) by age, sex, country, year, external cause of injury, and type of injury.

\section{Modelling Strategy}

Separate models were undertaken for injuries that necessitated inpatient care and injuries that required other health care. Injuries warranting inpatient care were cases that were serious enough to necessitate inpatient care, assuming there were no constraints on access to health care. Injuries requiring other health care were those injuries that were not severe enough to require hospitalization, but required other types of health care, such as treatment in an emergency department. Injuries not severe enough to require some form of health care intervention were not included in this research. This study also attempted to include cases where some kind of health care would have occurred, if health care had been readily available. In several self-report surveys asking about injuries sustained in the past month or year, the participants were also asked whether they sought care and to provide the reasons if they had not. This information was used to include cases who did not access health care for financial or geographic reasons. Incidence for cause-of-injury categories, including TIs, was modelled using DisMod-MR 2.1, which is a meta-regression tool that creates estimates of disease incidence, prevalence, remission, and mortality. More details on the modelling strategy can be found in a previous publication. ${ }^{8}$

As a measure of premature mortality, years of life lost (YLLs) were calculated by subtracting age at death from the longest possible life expectancy for an individual of that age, as defined by the GBD 2017 standard life table ${ }^{11}$ and multiplying this by the number of deaths recorded. YLDs were produced by multiplying the number of cases 
of each adverse health outcome by the disability weight assigned to each of these outcomes. The disability weight indicates the severity of the health loss and ranges from 0 (equivalent to full health) to 1 (equivalent to death). In addition, YLLs and YLDs were combined to form DALYs. All estimates were reported as count and agestandardized rates, which were estimated through the direct standardization method using the GBD standard population. ${ }^{8}$

We also determined the ratio of observed to expected, based on SDI, burden of TIs for the 22 EMR countries in 1990 and 2017. SDI is a summary measure of sociodemographic status and is composed of the lag distributed income per capita, mean educational attainment for those aged 15 and older, and the total fertility rate for those younger than $25 .{ }^{12}$ The SDI ranges from 0 (least developed) to 1 (most developed). 95\% uncertainty intervals (UIs) accompanied all estimates, in order to reflect the uncertainty arising from the raw data and data adjustments, as well as statistical modelling. ${ }^{12}$ GBD propagated uncertainty from all sources by repeating all calculations 1000 times, each time drawing from distributions, instead of point estimates, for all model parameters. $^{12}$

\section{Results}

Table 1 shows that in the EMR, there were 5531408 (UI 4895209-6213116) transport-related incidents in 2017. Pakistan had the largest number of incidents, with 1628711 (UI 1437527-1860201), while the lowest number of incidents was reported in Djibouti (8864, UI 7772-10048). The age-standardized incidence rate per 100000 for the EMR in 2017 was 787 (UI 705.5-876.2), which had not changed significantly from 1990 (-0.9\%; UI -4.7 to 3 ); it was remarkably different between countries, such that Oman (1303.9; UI 1167.3-1441.5) and Palestine
(486.5; UI 434.5-545.9) had the highest and lowest agestandardized incidence rates, respectively. Figure 1 shows the incident cases and incidence rates of TIs in the EMR by age group and sex. The graph shows that the total number of incidents involving males increased from the youngest age group (1-4 years old) and peaked in the 20-24 age group, before declining to the oldest age group $\left(95^{+}\right)$. A similar pattern was also shown for females, although the number of incidents involving females was much lower. This gap was particularly pronounced during late adolescence (1519 years old) and early-mid-adulthood (20-24 to 40-44 years old), but steadily reduced with increasing age until there were only small differences between sexes in the two oldest age groups (90-94 and 95').

Figure 1 shows that the incidence rate for males remained relatively stable across the first three age groups $(<1,1-4$ and 5-9 years old), and then reached a peak in the 25-29 age group. This rate remained relatively stable until the 55-59 age group, when a steep increase was observed over the remaining age groups. In contrast, the incidence rate for females remained relatively stable for the first three age groups ( $<1,1-4$ and 5-9 years old) and then showed a very slight increase to the 75-79 age group, which was followed by a substantial increase that reached a peak in the $95^{+}$age group.

Table 1 also shows that there were 185251 (UI $170840-$ $200635)$ transport-related deaths in the EMR in 2017, an increase from 140352 (UI 118673-156938) in 1990. In 2017, the age-standardized death rate per 100000 for the EMR was 29.5 (UI 27.1-31.9), which was 30.5\% lower than that found in 1990 (42.5; UI 36.8-47.3). Somalia (54; UI 30-77.4) had the highest age-standardized death rate per 100000 in 2017, while Lebanon (7.1; UI 4.8-8.6) had the lowest.

Figure 2 shows the number of deaths and death rates for TIs, by age group and sex. The pattern of results largely

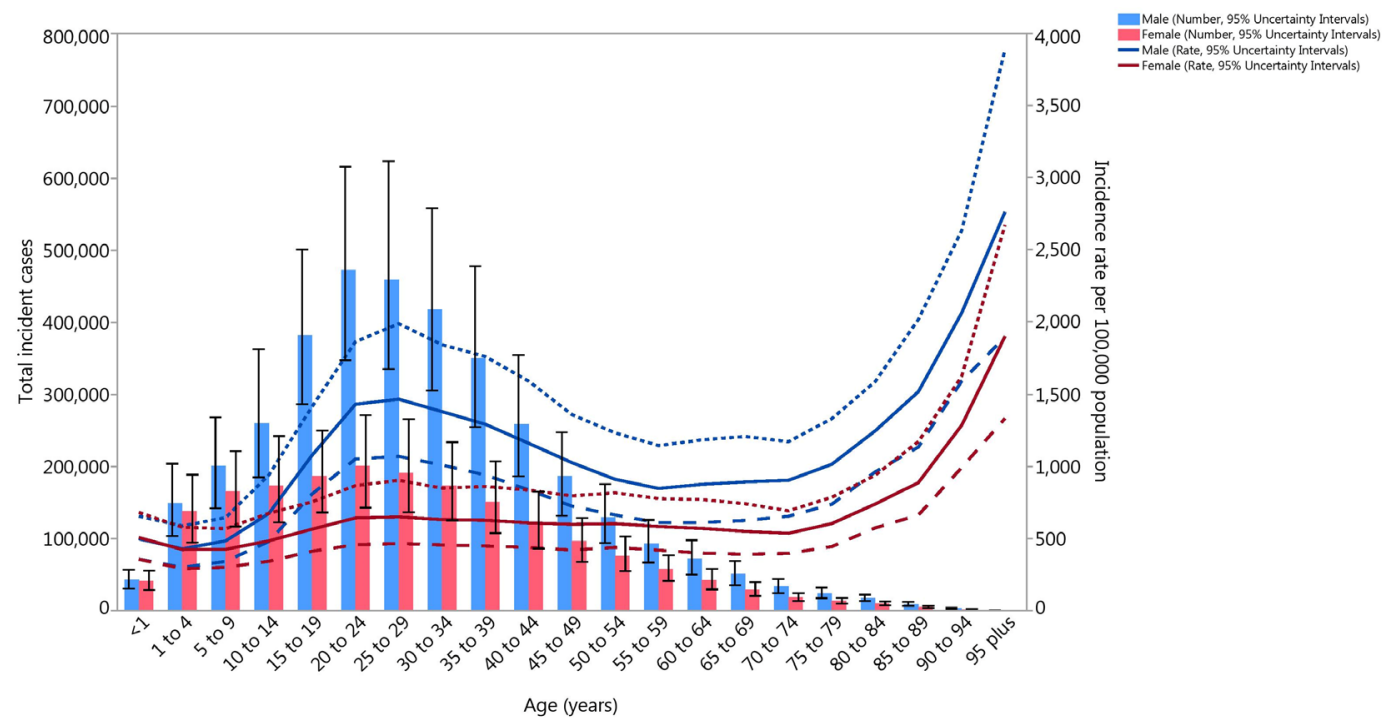

Figure 1. Number of Incident Cases and Incidence Rate of Transport-Related Injuries Per 100000 Population in the Eastern Mediterranean Region by Age and Aex, 2017. Dotted and dashed lines indicate 95\% upper and lower uncertainty intervals, respectively. 


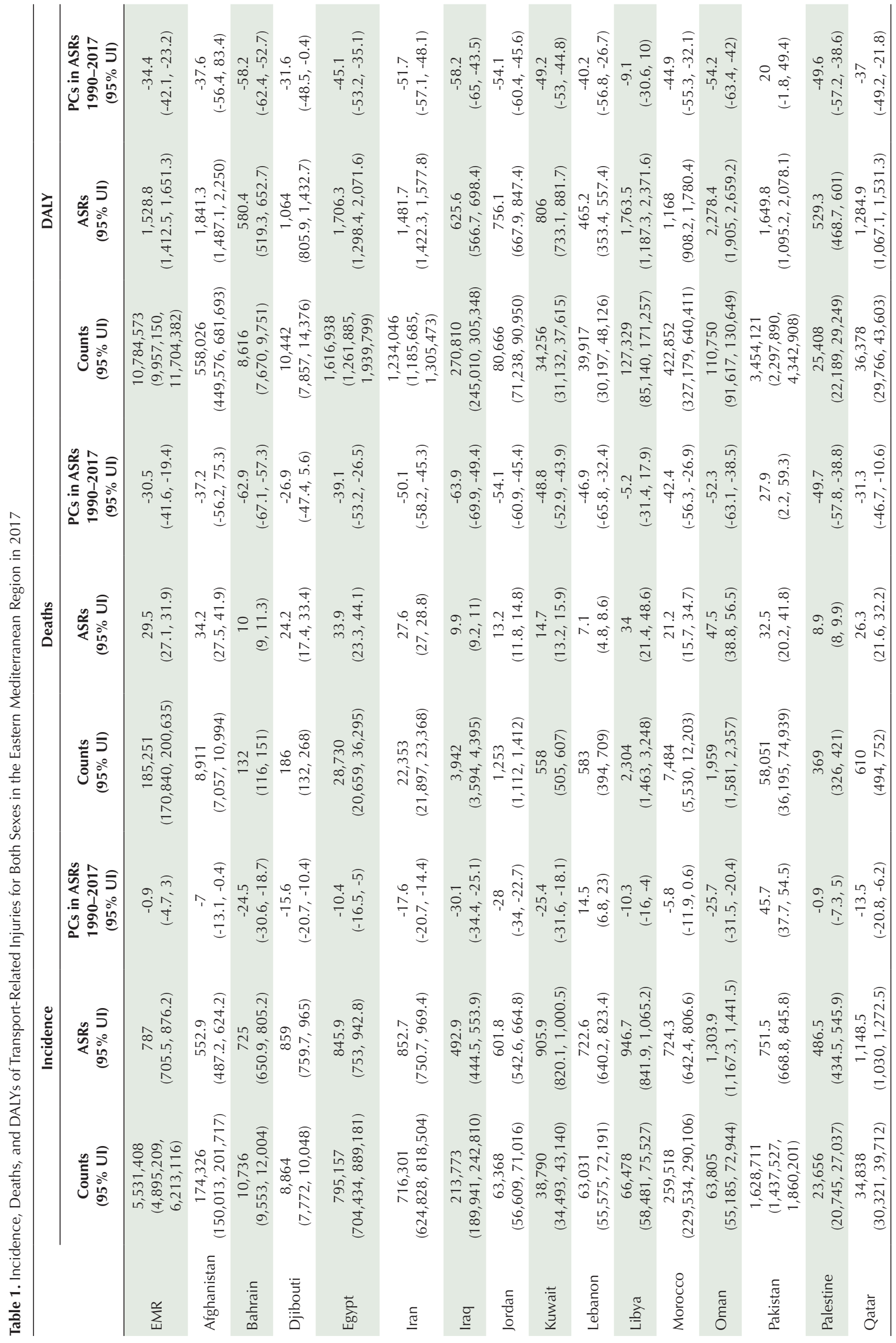




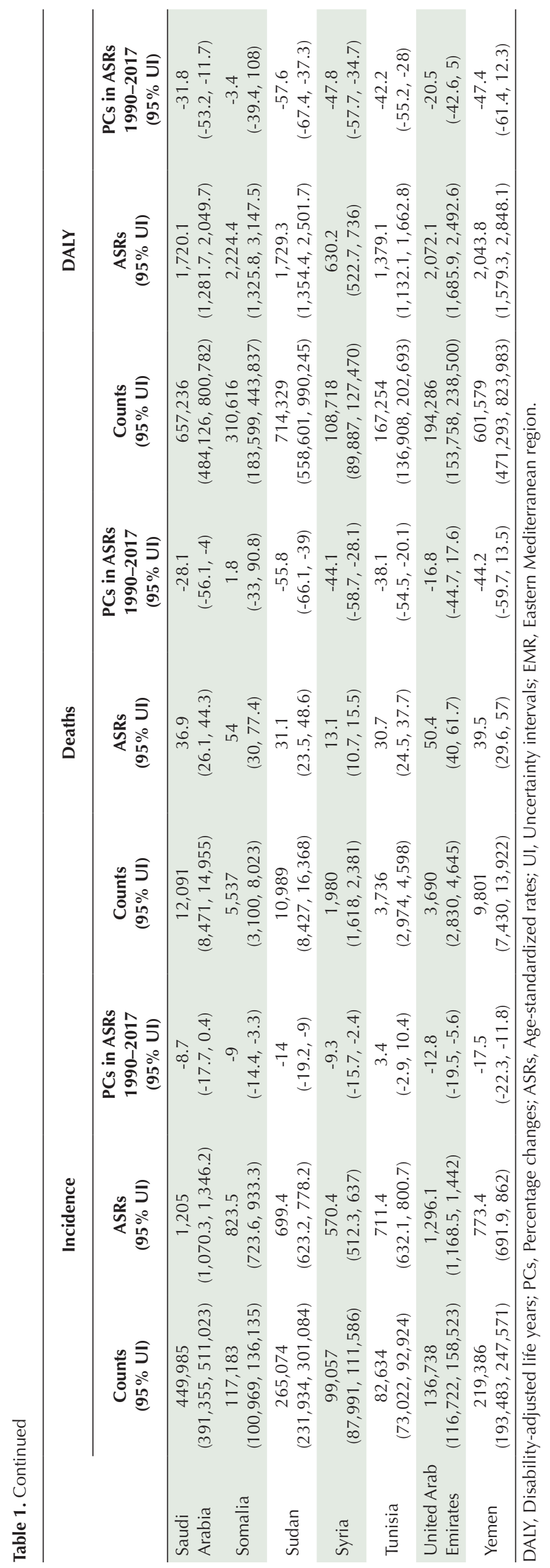


mirrors the incident cases and incidence rates, with a clear increase in the total number of deaths involving males after the four youngest age groups $(<1,1-4,5-9$, and 10-14 years old), which reached a peak in the $20-24$ age group and then declined until the $95^{+}$age group. A similar pattern was also found for females, albeit with a smaller number of deaths than for males. This gap was again particularly pronounced during late adolescence and early to mid-adulthood (15-19 to 40-44 years old), but steadily reduced with increasing age until there were only small differences between sexes in the two oldest age groups (90-94 and 95+).

The death rate for males remained relatively stable across the first four age groups $(<1,1-4,5-9$, and $10-14$ years old) but increased to a peak in the 20-24 age group (Figure 2). This rate remained relatively stable until the 55-59 age group, when an increasingly steep increase was observed over the remaining age groups. In contrast, the death rate for females decreased slightly across the first four age groups $(<1,1-4,5-9$, and 10-14 years old) and then showed a very slight increase until the 75-79 age group, after which a substantial increase occurred that reached a peak at the $95+$ age group.

In 2017, Somalia had the highest death rate in the region (54, UI 30-77.4), followed by the UAE (50.4, UI 40-61.7) and Oman (47.5, UI 38.8-56.5) (Table 1 and Figure 3). The lowest mortality rates were found in Lebanon (7.1, UI 4.8-8.6) and Palestine (8.9, UI 8-9.9). For all EMR

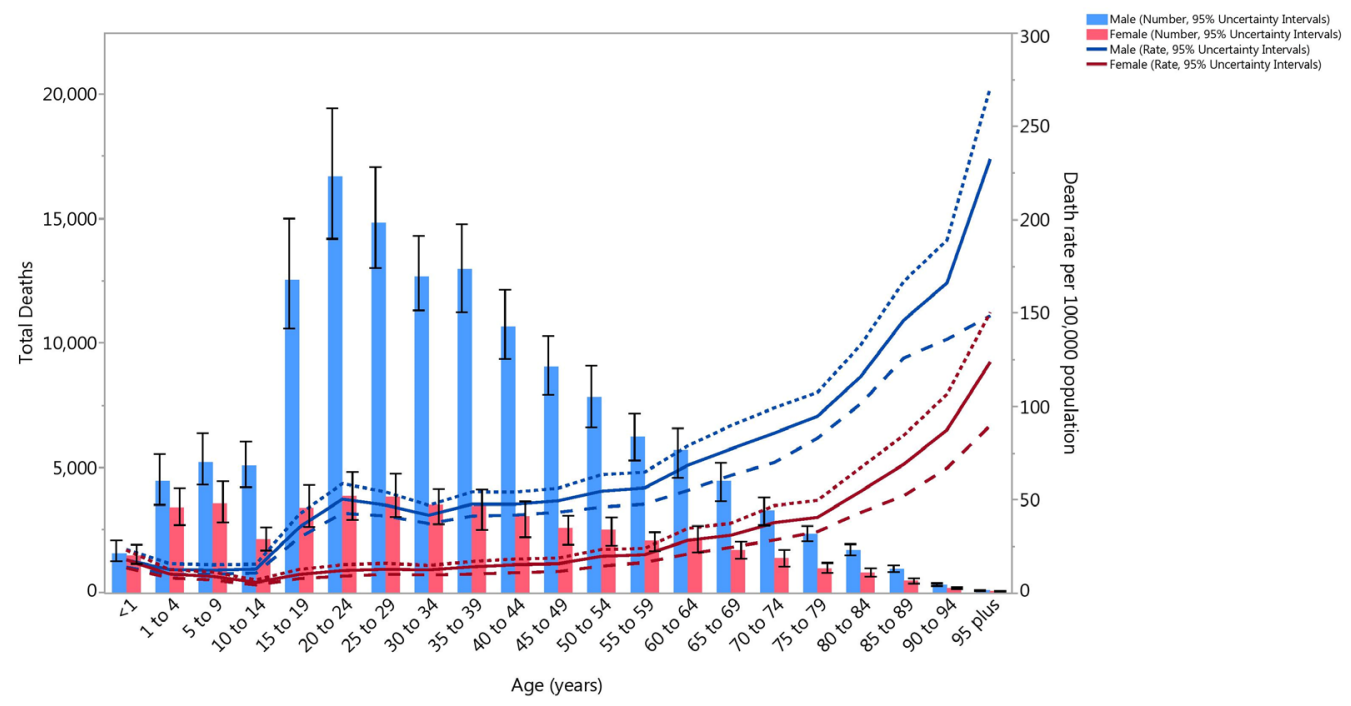

Figure 2. Number of Deaths and Death Rate of Transport-Related Injuries Per 100000 Population in the Eastern Mediterranean Region by Age and Sex, 2017. Dotted and dashed lines indicate 95\% upper and lower uncertainty intervals, respectively.

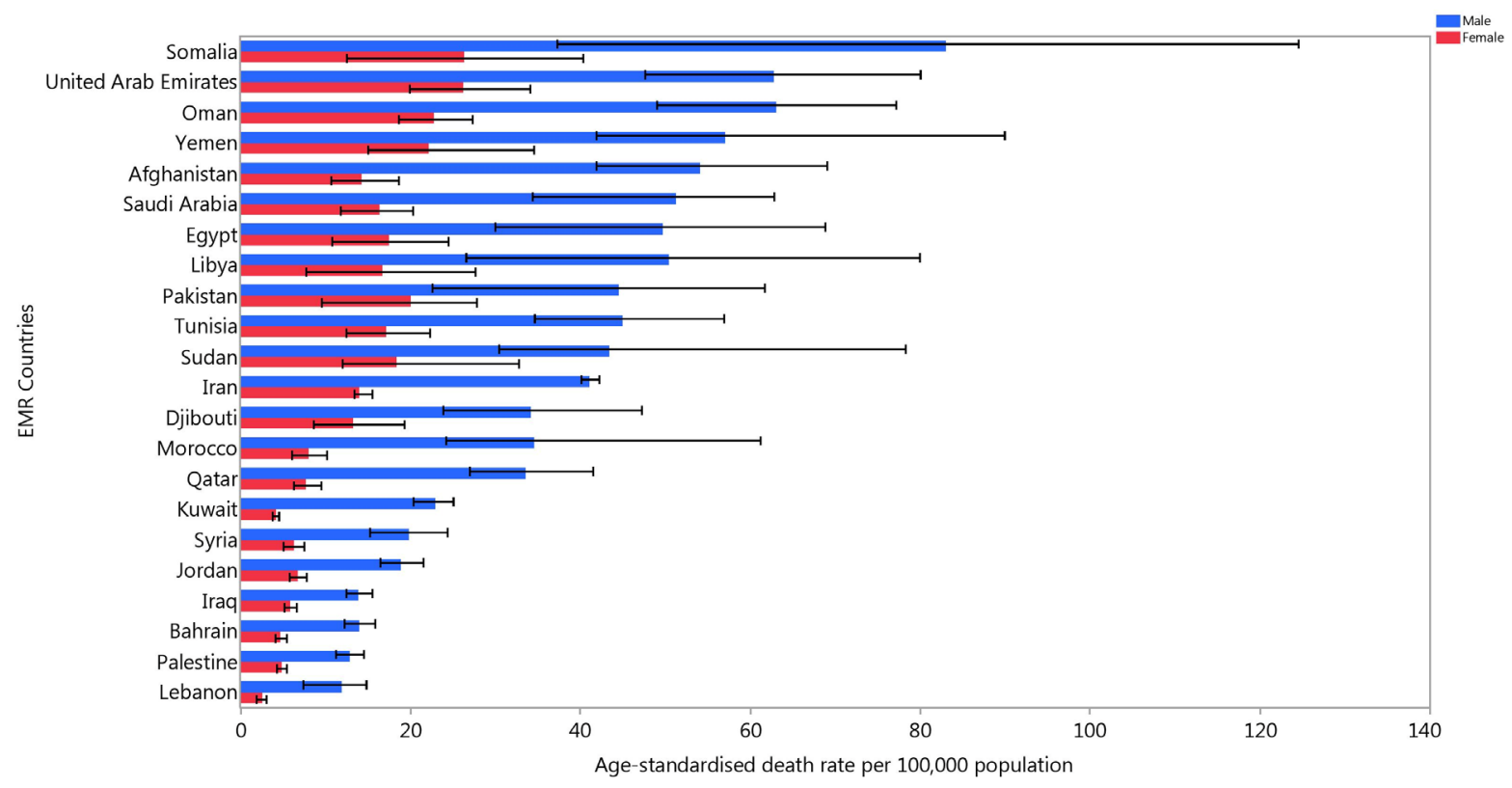

Figure 3. Age-Standardized Death Rates of Transport-Related Injuries in 2017 for the 22 Countries of the Eastern Mediterranean Region by Sex. 
countries, the death rates were considerably higher among males than females. The overall death rate in the region was 29.5 (UI 27.1-31.9). In 2017, the individual rate for males in the EMR was 42.3 (UI 38.5-46.1), while for females, this figure was 15.7 (UI 12.8-17.7). In the EMR, the age-standardized mortality ratio between males and females was 2.68. Within the EMR region, Kuwait had the highest ratio (5.5), followed by Lebanon (4.7). In contrast, the country with the lowest ratio was Pakistan, at 2.2.

The EMR overall showed a $30.5 \%$ (UI 19.4-41.6) reduction in age-standardized death rates since 1990 (Table 1). However, two countries, Pakistan (27.9\%, UI 2.2-59.3) and Somalia (1.8\%, UI -33 to 90.8) showed increases in the age-standardized death rates from 1990 to 2017, although it was not statistically significant for Somalia. All remaining countries showed a decrease in age-standardized death rates since 1990, with the largest decreases found in Iraq (-63.9\%, UI -69.9 to -49.4$)$ and Bahrain (-62.9\%, UI -67.1 to -57.3$)$.

Figure 4 shows the percentage change in agestandardized death rates by country and sex from 1990 to 2017. This shows that Pakistan was the only EMR country to register increases for both males and females, while Somalia registered an increase for males and a decrease for females. The remaining countries registered large reductions in age-standardized death rates since 1990. Furthermore, in most cases, a larger reduction in death rate was found for females than for males.

Figure 5 shows the percentage of deaths due to TIs by country and sub-causes in 2017. Pedestrian road injuries accounted for the largest part of the fatalities in Somalia (53.2\%) and Pakistan (45.1\%). In contrast, motor vehicle

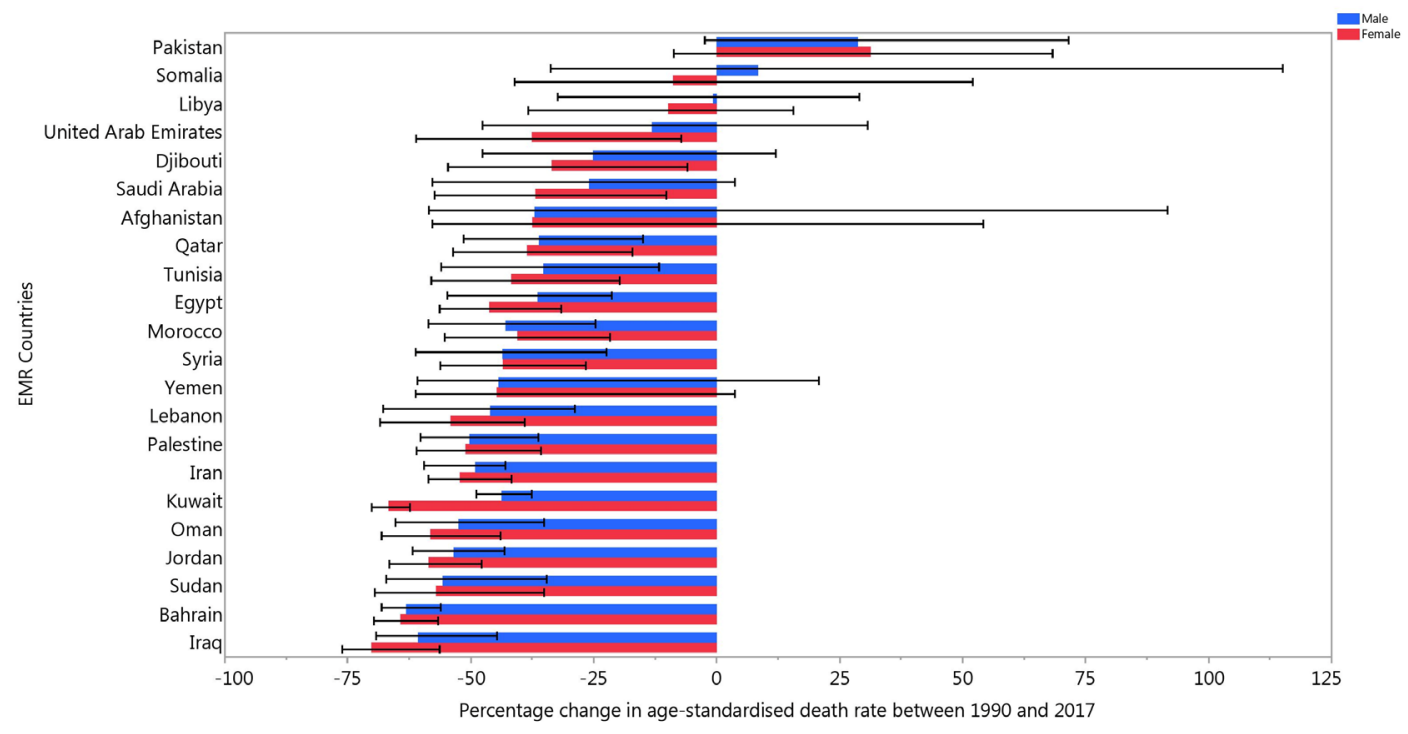

Figure 4. Percentage Change in Age-Standardized Death Rates of Transport-Related Injuries from 1990 to 2017 for the 22 Countries of the Eastern Mediterranean Region by Sex

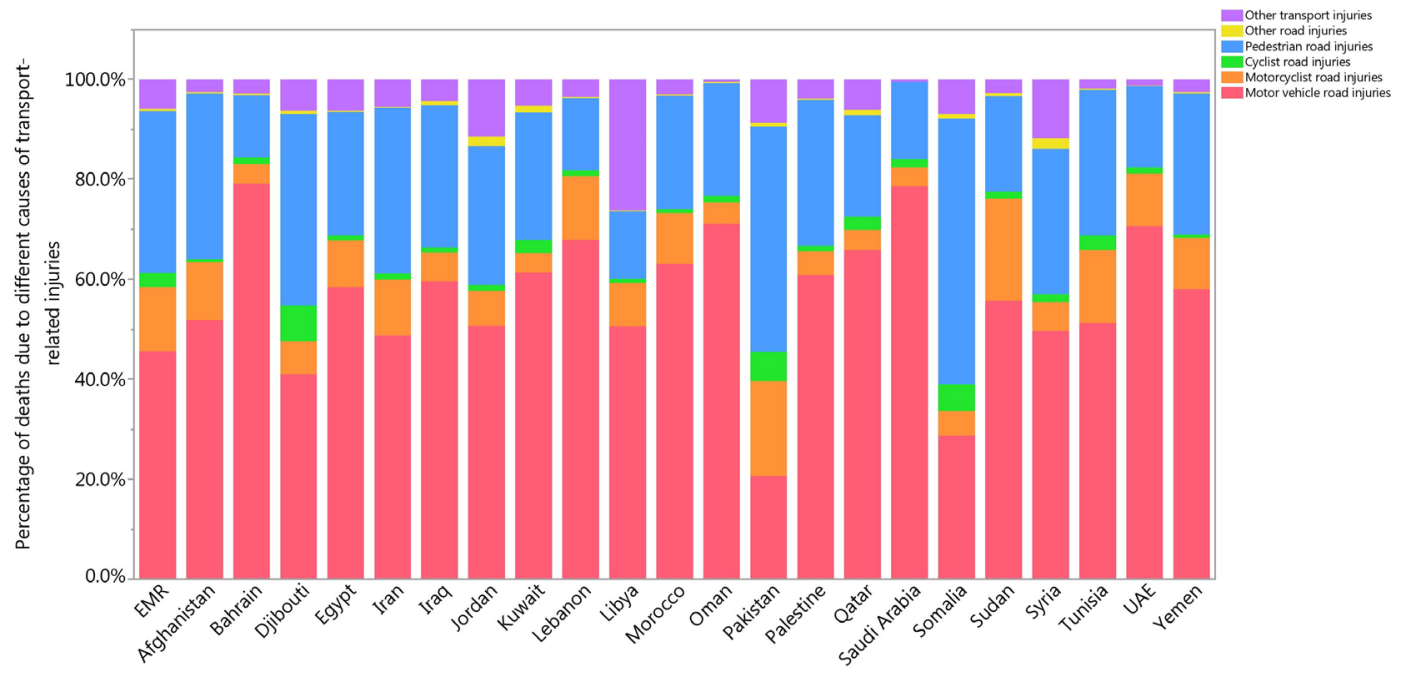

Figure 5. Percentage of Deaths due to Different Sub-causes of Transport-Related Injuries in 2017 for Both Sexes in the 22 Countries of the Eastern Mediterranean Region. 
road injuries were the dominant cause in the remaining countries.

Figure S1 shows the number and age-standardized rates for the sub-causes of TIs by age group in 2017. For the first three age groups $(<1,1-4$, and 5-9 years old), other TIs accounted for the largest number of incidents, while motor vehicle road injuries were the most common subcause until the 70-74 age group. The age-standardized rate for motor vehicle road injuries increased with age until the 30-34 age group and then slowly declined until the 70-74 age group. Also notable was the change in rate for other transport injuries, which declined across the first three age groups $(<1,1-4$, and 5-9 years old) before remaining relatively stable until the 65-69 age group and then rapidly increasing to peak in the $95+$ age group.

Figure 6 presents the number of deaths and death rates for the sub-causes of TIs in 2017. Pedestrian road injuries accounted for the largest number of deaths in three of the four youngest age groups (1-4, 5-9 and 10-14 years old) and also among the seven oldest age groups (from 65-69 to $95^{+}$). In the remaining age groups, motor vehicle road injuries were the largest cause of death, followed by injuries sustained by pedestrians and in motorcycle accidents. Figure 6 also shows that the death rates for motor vehicle road injuries steadily increased from the 15-19 age group, with a marked increase after the 80-84 age group. Fatalities sustained by pedestrians followed a similar pattern to motor vehicle road injuries at the start but were lower until the 65-69 age group, when they became higher than the rate of motor vehicle road injuries. The highest rates for both injury sub-causes were found in the 95+ age group. This pattern was generally observed at the country level, and the highest rates were observed in the oldest age group $\left(95^{+}\right.$years $)$, especially in the UAE, Somalia, and Oman (Figure 7).

\section{Years of Life Lost and Years Lived with Disability}

The number of YLLs and the age-standardized YLL rates

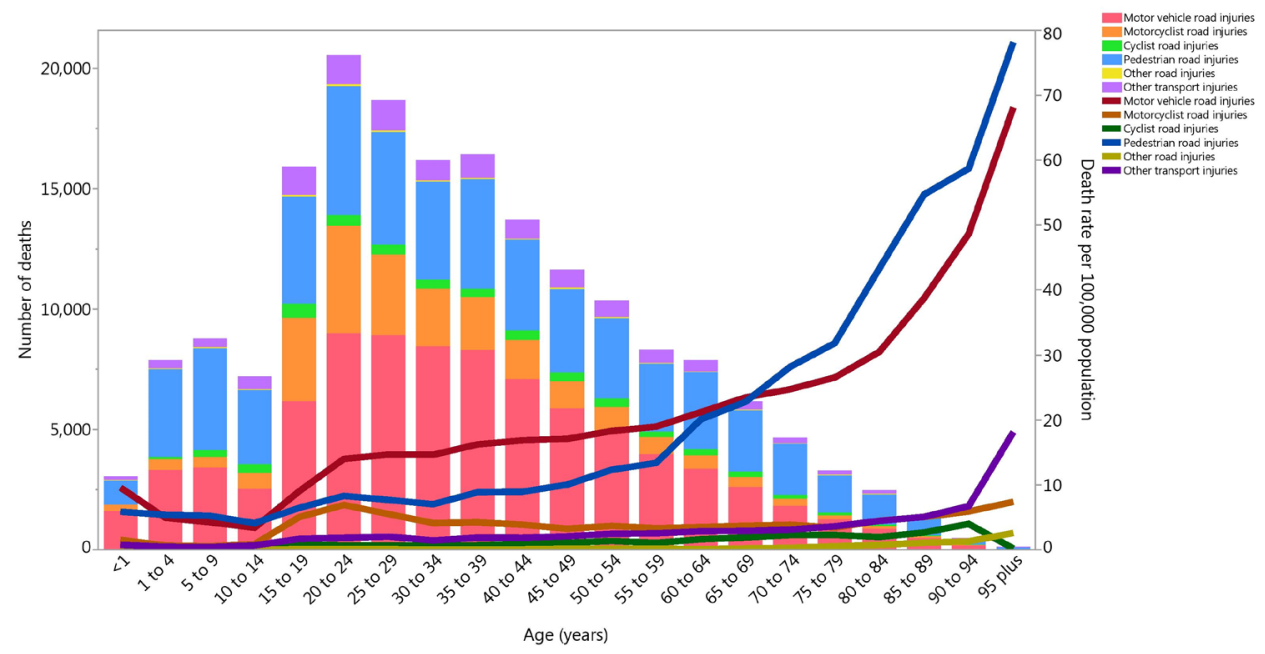

Figure 6. Number of Deaths and Death Rate of Transport-Related Injuries Per 100000 Population in the Eastern Mediterranean Region by Age and Sub-cause, 2017.

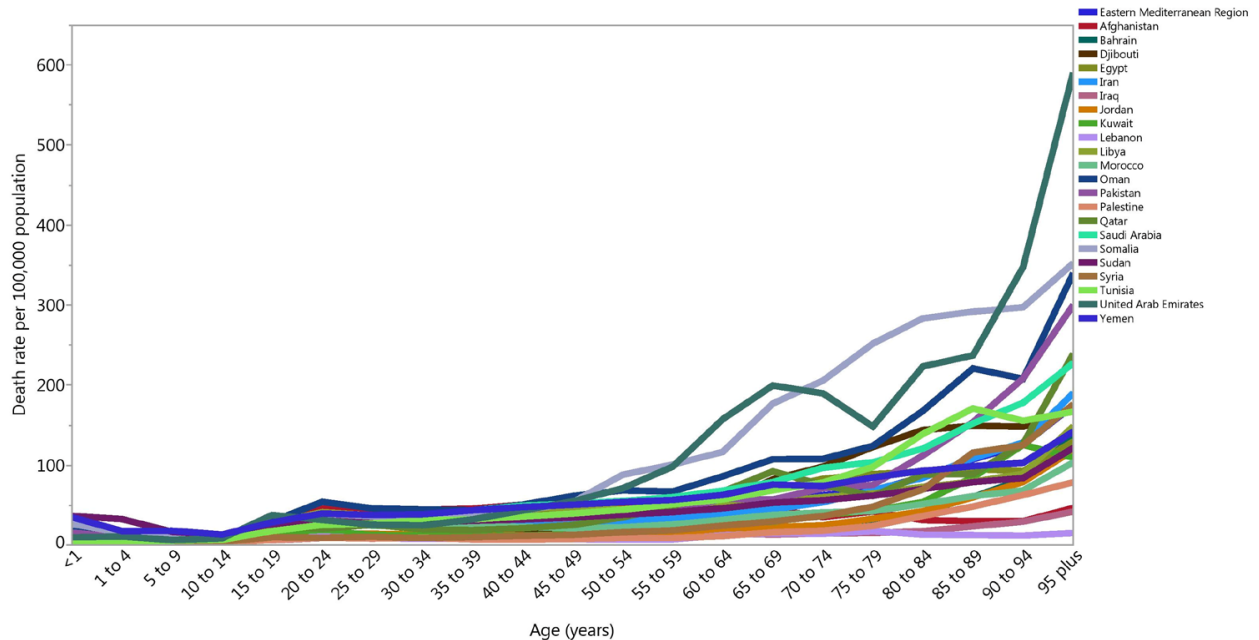

Figure 7. 2017 Death Rate of Transport-Related Injuries Per 100000 Population for the 22 Countries of the Eastern Mediterranean Region by Age. 
for the EMR in 2017 are presented in Figure S2, along with the number of YLDs and the age-standardized YLD rates. Figure S2 shows that the age-standardized YLD rate steadily increased with increasing age, while the agestandardized YLL rate showed a less consistent pattern by age group. The YLL rate decreased over the first three age groups (1-4, 5-9, 10-14 years old), then increased until the 20-24 age group and then decreased across the remaining age groups.

\section{Disability-Adjusted Life Years}

In 2017, the age-standardized DALY rate for the EMR was 1,528.8 (UI 1412.5-1651.3), which was 34.4\% lower than that found in 1990 (2331.3; UI 1993.1-2589.9) (Table 1). The highest DALY rates were found in Oman (2278.4; UI 1905.0-2659.2), UAE (2,072.1, UI 1685.9-2492.6), and Yemen (2043.8, UI 1579.3-2848.1), while the lowest were found in Lebanon (465.2, UI 353.4-557.4) and Palestine (529.3, UI 468.7-601) (Table 1). Figure S3 also shows that in all countries, males had much higher DALY rates than females, with the highest ratios in Kuwait (4.2), followed by Morocco (3.8) and Qatar (3.4).

The percentage change in age-standardized DALY rates from 1990 to 2017, by country and sex, is shown in Figure S4. Pakistan was the only country to show an increase in the age-standardized DALY rates for both sexes from 1990 to 2017, while Somalia showed an increase for males and a decrease for females. In contrast, all other countries registered a large decrease in DALY rates, with six countries (Bahrain, Iran, Iraq, Jordan, Oman and Sudan) recording an overall reduction of more than 50\% (Table 1). Furthermore, in most countries, a bigger reduction in the age-standardized DALY rate was observed for females (than males), except in Afghanistan, Qatar, Morocco, Syria, and Bahrain (Figure S4).

Figure S5 shows the number of DALYs and the DALY rate by sub-cause and age. The largest number of DALYs in the first three age groups was from injuries sustained by pedestrians, but for the older age groups, motor vehicle road injuries composed the majority of the DALYs, with a peak in the 20-24 age group. Figure S5 also shows that in late adolescence/early adulthood (15-19 to 30-34 years old), DALYs caused by motorcycles were also a large sub-cause of DALYs. This pattern is also reflected in the DALY rate for motorcycle-related injuries, which shows a dramatic increase in the 15-19 and 20-24 age groups, followed by a steady decline with age. The DALY rate caused by pedestrian road injuries decreased over the first three age groups and then increased until the 60-64 age group, before declining over the remaining age groups. The DALY rate for motor vehicle road injuries demonstrated a substantial decline for the first four age groups $(<1,1-4$, 5-9, and 10-14 years old), a steep increase in the 15-19 and 20-24 age groups, followed by a relatively constant decline to the $95+$ category. The DALY rate for motor vehicles was above that of pedestrian road injuries during most of adulthood but slipped below pedestrian road injuries in the 70-74 age group.

It was found that about half of the EMR countries, including the UAE, Oman, Saudi Arabia, Libya, Iran, Egypt, Tunisia, Yemen, Sudan, Somalia, and Qatar, had a higher than expected burden of TIs in 1990 and 2017 for both males and females (Figure 8).

\section{Discussion}

The current study shows that the absolute number of TI-related deaths has increased from 140352 in 1990 to 185251 in 2017. Meanwhile, the incidence rate has remained at almost the same level ( $0.9 \%$ decrease), while death $(-30.5 \%)$ and DALY $(-34.4 \%)$ rates have decreased substantially. Thus, the relative development in traffic safety has been positive, while the absolute cost of transport, in terms of loss of lives and human suffering, has increased. The relative improvement of safety in the

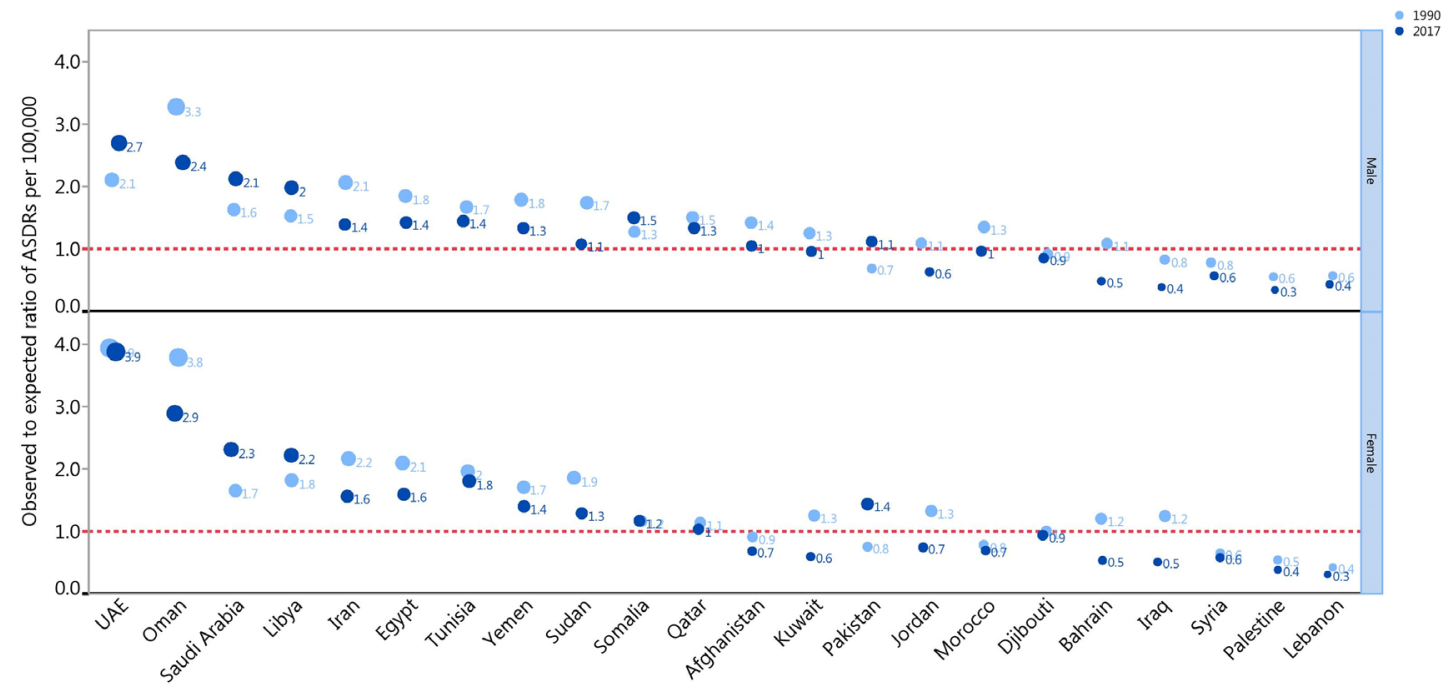

Figure 8. Observed to Expected Ratio of Age-Standardized Death Rates of Transport-Related Injuries Per 100000 Population for the 22 Countries of the Eastern Mediterranean Region by Sex, 1990 and 2017. 
EMR can be explained partly by the level of motorization as Smeed's law proposes: increasing traffic volume can lead to increase in the absolute number of fatalities, but a decrease in fatalities per vehicle. ${ }^{13,14}$ It should be noted that Western industrialized countries show clear declines in both absolute number of road deaths and the number of deaths per capita or per registered vehicles/kilometers driven. ${ }^{5}$ Thus, urgent actions are needed in the EMR to reverse the trend of increasing transport-related deaths.

The results of this research show that in terms of traffic safety, the EMR is not a homogeneous area. The lowest level of traffic safety can be observed in Somalia, with an age-standardized death rate of 54.0, while the safest traffic in the region can be found in Lebanon, with an agestandardized death rate of 7.1 (i.e., road traffic is 7.6 times riskier in Somalia than in Lebanon). Almost the same ratio can also be found when comparing the DALY rates of Somalia (2224.4) and Lebanon (465.2). Interestingly, large differences can also be seen in the percentage changes in age-standardized death rates and DALY rates: some countries show sharp declines in deaths and DALYs rates (Iraq, Bahrain, Sudan), while in other countries, the decline has been much more modest (e.g., Libya, the UAE), or the rates have even increased (Pakistan, Somalia). While the DALYs and death rates represent different ways of measuring the human cost of traffic injuries, these two measures seem to give a very similar picture of the phenomenon.

The large variation in the death figures and recent developments in traffic safety among EMR countries shows a remarkable degree of diversity among these countries. ${ }^{7}$ The change in transport-related fatalities statistics can be caused by a number of different factors, including motorization rate, ${ }^{15}$ population growth rate, urbanization, ${ }^{16}$ the general level of emergency services, ${ }^{17}$ differences in road infrastructure, and level of law enforcement. ${ }^{18}$ The fact that some of the wealthiest countries of the EMR (e.g., Saudi Arabia, the UAE) have much riskier traffic and show less development in safety underlines the fact that traffic safety is not necessarily linked to wealth or per capita income in a country. ${ }^{16}$ There are many other transportation-related and socioeconomic factors, which may explain why some countries are riskier than others. ${ }^{16}$ In the present study, age-standardized DALY rates were studied as a function of SDI scores. While the SDI was in general negatively related to age-standardized DALY rates, significant differences were found among the countries that compose the EMR. In Oman, for example, improved traffic safety, as a function of SDI, was deep and fairly linear, while in Pakistan, the DALY rate increased as a function of SDI. The results of the present study show that the relationship between socioeconomic development and traffic safety is far from simple and should be thoroughly investigated (Figure 8). Economic development does not necessarily result in improvements in traffic safety if a country does not allocate funds to traffic safety interventions.
The present study also investigated the burden of TIs and deaths in the EMR by age and sex. The results were in line with the results observed virtually everywhere: males were more likely to be involved in a traffic accident and to lose their lives in traffic. ${ }^{19,20}$ This was especially remarkable among young and middle-aged road users: the differences between males and females was the largest among the 20to 24-year-old, while the overrepresentation of males was much smaller among those under 15 years of age and the elderly ( $>75$ years old). These differences between males and females may reflect the general participation in traffic and work life between sexes: in countries where females work less outside home and thus, commute less than males, the differences between sexes in road causalities are likely to be more pronounced. When evaluating the proportion of different types of road deaths and DALYs, we can see that the share of motor vehicle road injuries is the highest among young adults, which reflects the fact that a large number of motor vehicle drivers are young males. In addition to exposure-related factors, higher transport-related death rates among young males are probably caused by risky behaviors, such as speeding, ${ }^{21}$ distracted driving, ${ }^{22}$ rule violations, ${ }^{23}$ driving while intoxicated, ${ }^{24}$ and not using a seatbelt, which are all more common among young males than other road user groups. ${ }^{20}$ The prevalence of these risky behaviors among young drivers is likely to reflect the traffic culture, level of enforcement, and level of driver education, which all vary to a large degree among EMR countries. Local policies and preventive interventions targeting these system and culture-level risk factors are urgently needed for reducing traffic fatalities in EMR countries. These interventions should be firmly rooted on sound research findings from investigations conducted globally, but especially in the target regions. The findings of the present study highlight the need for future research to address road injury as a substantial public health problem in the EMR.

As with GBD estimates for other diseases and injuries, data sparsity was the main limitation of the present study. Therefore, the burden of TIs for the countries with sparse data were modelled using country-level covariates and borrowing the data from similar EMR countries. The estimates for these countries were used along with wide UIs and should be interpreted with caution. To address this problem, ongoing data collection on TIs is encouraged, especially for countries with absent or insufficient data.

It should be noted that the accuracy and efficiency of data recording among EMR countries is expected to vary widely. Some countries have fully or close to fully digitalized injury data recording systems, while others still rely on manual recording of injury data. This might have created some biases in the data collected and the subsequent statistical analyses. At the same time, EMR countries with good legal records are expected to have better injury recording systems than countries engaged in some form of military conflict (like Syria and Afghanistan) or other forms of civil disorder (like Iraq). With the 
iterative methodology followed in the current study, it is thought that such differences would be minimized.

In addition, the long-term cohort studies were used for estimating the injury severity and the probability of long-term disability was available for limited number of countries and additional data in the future GBD cycles are needed to make the estimates more robust.

In conclusion, the present study shows that while road traffic has become relatively safer (measured by deaths and DALYs per 100000 population), the number of transport-related deaths in the EMR is growing and needs to be addressed urgently. Traffic incidents among young males are a large problem, which should be prioritized in traffic safety policies. The socioeconomic development of an EMR country might not necessarily lead to decreases in death and DALY rates. In fact, EMR countries with high income per capita still have a high risk for their road users despite the advancement in the road network and medical emergency services. This underdevelopment in road safety indicates that traffic safety is not necessarily linked to wealth or per capita income in a country, but involves other factors such as driving culture (which is demonstrated in unsafe driving habits like speeding, crossing red lights, sudden changes in lanes and others). Therefore, it is recommended that governments in those countries develop short- and long-term plans to change this driving culture in order to reduce the risk on their roads.

\section{Authors' Contribution}

SS, MJMS, TL, and TH prepared the first draft. SS and MN provided overall guidance. SS and MN managed the project. SS and AAH analyzed data. SS, MJMS, TL, and TH finalized the manuscript on the basis of comments from other authors and reviewer feedback. All other authors provided data, developed models, reviewed results, provided guidance on methods, or reviewed and contributed to the manuscript.

\section{Conflict of Interest Disclosures}

The authors have no conflicts of interest to declare.

\section{Ethical Statement}

The present study was approved by the Ethic Committee of Tabriz University of Medical Sciences (IR.TBZMED.REC.1397.1084).

\section{Acknowledgements}

Saeid Safiri acknowledges support from the Road Traffic Injury Research Center, Tabriz University of Medical Sciences, Tabriz, Iran (Grant No 62894). Syed Aljunid acknowledges the Department of Health Policy and Management, Faculty of Public Health at Kuwait University and the International Centre for Casemix and Clinical Coding, Faculty of Medicine at the National University of Malaysia for their approval and support to participate in this research project. Seyed Sina Naghibi Irvani would like to thank the Clinical Research Development Unit (CRDU) of Loghman Hakim Hospital, Shahid Beheshti University of Medical Sciences, Tehran, Iran for their support. Abdallah M. Samy acknowledges support from the Egyptian Fulbright Mission Program. Mohammadreza Sobhiyeh thank all staff of the peripheral intervention ward of Imam Reza Hospital for their cooperation in this study as well as the clinical research development center of Imam Reza Hospital, Kermanshah University of Medical Sciences for their wish advice.

\section{Supplementary Materials}

Supplementary file 1 contains Figures S1-S5.

\section{References}

1. World Health Organization. Global Health Estimates Available from: http://www.who.int/healthinfo/global_ burden_disease/en/. Accessed 14 June 2019.

2. World Health Organization (WHO). Global Status Report on Road Safety 2018. WHO; 2018.

3. Bachani AM, Zhang XJ, Allen KA, Hyder AA. Injuries and violence in the Eastern Mediterranean Region: a review of the health, economic and social burden. East Mediterr Health J. 2014;20(10):643-52.

4. GBD 2017 Population andFertilityCollaborators. Population and fertility by age and sex for 195 countries and territories, 1950-2017: a systematic analysis for the Global Burden of Disease Study 2017. Lancet. 2018;392(10159):1995-2051. doi: 10.1016/s0140-6736(18)32278-5.

5. World Health Organization (WHO). Global Status Report on Road Safety 2015. Geneva: WHO; 2015.

6. GBD 2015 Eastern Mediterranean Region Transportation Injuries Collaborators. Transport injuries and deaths in the Eastern Mediterranean Region: findings from the Global Burden of Disease 2015 Study. Int J Public Health. 2018;63(Suppl 1):187-98. doi: 10.1007/s00038-017-0987-0.

7. Sengoelge M, Laflamme L, El-Khatib Z. Ecological study of road traffic injuries in the eastern Mediterranean region: country economic level, road user category and gender perspectives. BMC Public Health. 2018;18(1):236. doi: 10.1186/s12889-018-5150-1.

8. GBD 2017 Causes of Death Collaborators. Global, regional, and national age-sex-specific mortality for 282 causes of death in 195 countries and territories, 1980-2017: a systematic analysis for the Global Burden of Disease Study 2017. Lancet. 2018;392(10159):1736-88. doi: 10.1016/ s0140-6736(18)32203-7.

9. GBD 2017 Disease and Injury Incidence and Prevalence Collaborators. Global, regional, and national incidence, prevalence, and years lived with disability for 354 diseases and injuries for 195 countries and territories, 1990-2017: a systematic analysis for the Global Burden of Disease Study 2017. Lancet. 2018;392(10159):1789-858. doi: 10.1016/ s0140-6736(18)32279-7.

10. GBD 2016 Risk Factors Collaborators. Global, regional, and national comparative risk assessment of 84 behavioural, environmental and occupational, and metabolic risks or clusters of risks, 1990-2016: a systematic analysis for the Global Burden of Disease Study 2016. Lancet. 2017;390(10100):1345-422. doi: 10.1016/s01406736(17)32366-8.

11. GBD 2017 Mortality Collaborators. Global, regional, and national age-sex-specific mortality and life expectancy, 1950-2017: a systematic analysis for the Global Burden of Disease Study 2017. Lancet. 2018;392(10159):1684-735. doi: 10.1016/s0140-6736(18)31891-9.

12. GBD 2017 DALYs and HALE Collaborators. Global, regional, and national disability-adjusted life-years 
(DALYs) for 359 diseases and injuries and healthy life expectancy (HALE) for 195 countries and territories, 1990-2017: a systematic analysis for the Global Burden of Disease Study 2017. Lancet. 2018;392(10159):1859-922. doi: 10.1016/s0140-6736(18)32335-3.

13. Smeed RJ. Some statistical aspects of road safety research. J R Stat Soc Ser A. 1949;112(1):1-34. doi: 10.2307/2984177.

14. Ponnaluri RV. Modeling road traffic fatalities in India: Smeed's law, time invariance and regional specificity. IATSS Res. 2012;36(1):75-82. doi: 10.1016/j.iatssr.2012.05.001.

15. Zhang J, Garrick NW, Atkinson-Palombo C, Ahangari H. How similar is China's traffic safety pattern to industrialized countries in their early stage of motorization? an analysis of death registration data for large and small/medium cities. Journal of Transportation Safety \& Security. 2017;9(Suppl 1):83-102. doi: 10.1080/19439962.2016.1201876.

16. Bener A, Yousif A, Al-Malki MA, El-Jack I, Bener M. Is road traffic fatalities affected by economic growth and urbanization development? Adv Transp Stud. 2011(23):8996. doi: 10.4399/97888548388648.

17. Ma L, Zhang H, Yan X, Wang J, Song Z, Xiong H. Smooth associations between the emergency medical services response time and the risk of death in road traffic crashes. J Transp Health. 2019;12:379-91. doi: 10.1016/j. jth.2018.08.011.

18. Solmazer G, Üzümcüoğlu Y, Özkan T. The role of traffic law enforcements in the relationship between cultural variables and traffic fatality rates across some countries of the world. Transp Res Part F Traffic Psychol Behav. 2016;38:137-50. doi: 10.1016/j.trf.2016.01.001.

19. Evans L. Traffic Safety. Bloomfield Hills, MI: Science Serving Society; 2004.

20. Shinar D. Traffic Safety and Human Behavior. Bingley: Emerald Group Publishing; 2017.

21. Wickens CM, Mann RE, Stoduto G, Ialomiteanu A, Smart RG. Age group differences in self-reported aggressive driving perpetration and victimization. Transp Res Part F Traffic Psychol Behav. 2011;14(5):400-12. doi: 10.1016/j. trf.2011.04.007.

22. Hoff J, Grell J, Lohrman N, Stehly C, Stoltzfus J, Wainwright $\mathrm{G}$, et al. Distracted driving and implications for injury prevention in adults. J Trauma Nurs. 2013;20(1):31-4. doi: 10.1097/JTN.0b013e318286616c.

23. Yagil D. Gender and age-related differences in attitudes toward traffic laws and traffic violations. Transp Res Part F Traffic Psychol Behav. 1998;1(2):123-35. doi: 10.1016/ s1369-8478(98)00010-2.

24. Fan AZ, Grant BF, Ruan WJ, Huang B, Chou SP. Drinking and driving among adults in the United States: results from the 2012-2013 national epidemiologic survey on alcohol and related conditions-III. Accid Anal Prev. 2019;125:4955. doi: 10.1016/j.aap.2019.01.016. 